\section{References}

Bigg, E. K. (1964), Influence of the satellite Io on Jupiter's decametric emission, Nature 203, 1008-1010.

Booker, H. G. (1962), Guidance of radio and hydromagnetic waves in the magnetosphere, J. Geophys. Res. 67, 4135-4162.

Carr, T. D., A. G. Smith, H. Bollhagen, N. F. Six, and N. E. Chatterton (1961), Recent decameter-wavelength observations of Jupiter, Saturn, and Venus, Astrophys. J. 134, 105-125.

Carr, T. D. (1962), The possible role of field-alined ducts in the escape of decameter radiation from Jupiter, presented at NASA Jupiter conference, New York.

Carr, T. D., G. W. Brown, A. G. Smith, C. S. Higgins, H. Bollhagen, J. May, and J. Levy (1964), Spectral distribution of the decameter radiation from Jupiter in 1961, Astrophys. J. 140, 778-795.

Dowden, R. L. (1963), Polarization measurements of Jupiter radio bursts at $10.1 \mathrm{Mc} / \mathrm{s}$, Australian J. Phys. 16, 398 .

Ellis, G. R. A., and P. M. McCulloch (1963), The decametric radio emissions of Jupiter, Australian J. Phys. 16, 380-397.

Gulkis, S. (1965), A theoretical model for the emission of the decametric radiation from Jupiter, Ph.D. dissertation, University of Florida.

Lebo, G. R., A. G. Smith, and T. D. Carr (1965), Jupiter's decametric emission correlated with the longitudes of the first three Galilean satellites, Science 148, 1724-1725.
Piddington, J. H. (1960), Radio Astronomy (Harper and Brothers, New York).

Smith, A. G., G. R. Lebo, N. F. Six, T. D. Carr, H. Bollhagen, J. May, and J. Levy (1965), Decameter-wavelength observations of Jupiter: the apparitions of 1961 and 1962, Astrophys. J. 141, 457-477.

Warwick, J. W. (1963), Dynamic spectra of Jupiter's decametric emission, 1961, Astrophys. J. 137, 41-60.

Yabroff, I. (1961), Computation of whistler ray paths, J. Res. NBS 65D (Radio Prop.), 485-505.

\section{Discussion Following Carr et al.'s Paper}

J. A. Roberts: Are the other satellites over the same part of Jupiter as Io when the low-frequency emission is stimulated?

Answer: Probably not.

\title{
Results From CSIRO, Sydney, Australia
}

\author{
O. B. Slee and C. S. Higgins \\ Radiophysics Laboratory, Commonwealth Scientific and Industrial Research Organization, \\ Sydney, Australia
}

The CSIRO data at $19.7 \mathrm{Mc} / \mathrm{s}$ confirm in general terms the influence of Io reported by Bigg [1964].

The major recent result has been the measurement of the angular size of the source of the bursts at $19.7 \mathrm{Mc} / \mathrm{s}$. This continues earlier work [Slee and Higgins, 1963] in which the sources were not significantly resolved. The present observations were made near the oppositions of 1963 and 1964, using baselines up to 12,700 wavelengths long. At the longest baselines the sources are well resolved (fig. 1), the apparent diameters ranging from $5 \mathrm{sec}$ of arc to greater than $15 \mathrm{sec}$ of arc. This variability is real.

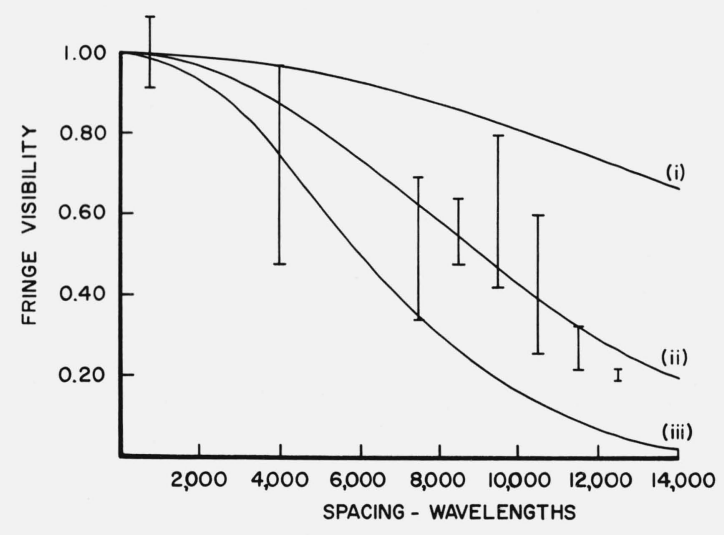

FigurE 1. Ranges of observed Jovian fringe visibility plotted against effective spacing of the interferometer. The curves represent the theoretical fringe visibilities for Gaussian sources of half-brightness sizes (i) $5 \mathrm{sec}$ of arc, (ii) $10 \mathrm{sec}$ of arc, and (iii) $15 \mathrm{sec}$ of arc. 
At periods when the bursts are similar at the two ends of the baseline, the fringes are stable over periods of several minutes. In a typical example, the 42 fringes recorded over a period of several minutes indicated a systematic movement of less than $4 \mathrm{sec}$ of arc, and a maximum scatter of $\pm 3 \mathrm{sec}$ of arc. Thus neither the size of the region in which successive bursts occurred, nor its systematic movement, exceeded 0.1 of the planetary diameter.

Observations of the increase in the angular size of other radio sources seen through the ecliptic suggest that interplanetary scattering can explain the observed angular sizes of the Jupiter bursts, and that the intrinsic size of the bursts is less than the apparent size. It is suggested that the scattering regions are 0.01 to $4.2 \mathrm{~A}$.U. from the Earth. It is furthermore suggested that the apparent burst structure of the Jovian radiation is produced by scintillations occurring within 0.01 A.U. of the Earth.

Note added in proof: The authors now consider that the restriction of the electron irregularities responsible for the scintillations to a region within 0.01 A.U. of the Earth is probably not correct on account of focusing difficulties. In all probability, the observed angular sizes and the burstiness of the radiation are manifestations of a random diffraction process occurring between the Earth and Jupiter, although it is not certain that the same electron irregularities are responsible for both effects.

\title{
References
}

Bigg, E. K. (1964), Influence of the satellite Io on the Jupiter's decametric emission, Nature 203, No. 4949, 1008-1010. Slee, O. B., and Higgins, C. S. (1963), Long baseline interferometry of Jovian decametric radio bursts, Nature 197, 781-782.

\section{Discussion Following Slee and Higgins' Paper}

Frank Drake: J. N. Douglas has recently shown that the direction of drifts observed over several stations reverses at times of Jupiter opposition. This reversal is consistent with the sources of the scintillations moving along with the solar wind outward from the Sun. The inferred scale of structures is hundreds of kilometers, and the speeds are upwards of $1000 \mathrm{~km} / \mathrm{sec}$. This picture is consistent with our picture of interplanetary scintillations observed in small radio sources by Hewish.

\section{Frequency and Polarization Structure of Jupiter's Decametric Emission on a 10-Millisecond Scale}

\author{
James W. Warwick and Mark A. Gordon \\ Astro-Geophysics Department, University of Colorado \\ and
}

High Altitude Observatory, Boulder, Colo.

\begin{abstract}
We present observations of decameter Jupiter emission on a 10-millisecond time base. After describing the swept-frequency polarimeter we discuss polarization and spectral characteristics in terms of propagation conditions along the ray path from Jupiter to Arecibo.
\end{abstract}

Formidable evidence has accumulated during the 10 years of Jupiter decametric observations on the existence and reality of exceedingly short-lived and narrowband phenomena. On time scales from no longer than a few seconds down to a few milliseconds, these structures have been seen with a variety of different techniques, from dynamic spectrographs, through multistation equipment, to wide-baseline interferometers. The major question that has been asked has been the source of the fluctuations: Is it in the terrestrial ionosphere, in interplanetary space, or in or near the source of the radiation at Jupiter itself? The answer given generally has been that the interplanetary medium is responsible, the data thus suggesting fine structure in the solar wind plasma flowing out from the Sun. In fact some of the data (such as time shifts observed between data from multiple stations) support this conclusion, but others do not (for example, failure of correlation at closely spaced stations, and some of the structures on dynamic spectra).

With these problems in mind we decided to investigate the fast-time evolution of the spectrum with as complete a determination of the state of polarization 